\title{
EL BIENESTAR SOCIAL DE PRESERVAR LA EROSIÓN: LOS BADLANDS DE LA REGIÓN DE MURCIA
}

José A. Albaladejo-García ${ }^{a^{*}}$, Francisco Alcón ${ }^{b}$, José A. Zabala ${ }^{b}$, Víctor Martínez-García ${ }^{b}$, María I. Rodríguez-Valero $^{a}$, Erasmo López-Becerra ${ }^{b}$, María D. de-Miguel ${ }^{b}$, Rafaela Dios-Palomares ${ }^{c}$, José M. Martínez-Paz ${ }^{a}$

${ }^{a}$ Departamento de Economía Aplicada. Universidad de Murcia (Murcia, joseantonio.albaladejo@um.es, mariaisabel.rodriguez1@um.es, jmpaz@um.es).

b ETSIA. Universidad Politécnica de Cartagena (Cartagena, francisco.alcon@upct.es, joseangel.zabala@upct.es,victor.martinez@upct.es,_erasmo.lopez@upct.es,md.miguel@upct.es ).

c Departamento de Estadística e Investigación Operativa. Universidad de Córdoba (Córdoba, rdios@uco.es )

\section{Resumen}

Muchos de los ecosistemas de zonas áridas se caracterizan por una baja provisión de servicios ecosistémicos (SE) de abastecimiento y regulación y, a menudo, son percibidos como carentes de SE culturales. Los Badlands son sistemas áridos sin vegetación, característicos del sureste español, cuyo valor social ha sido escasamente estudiado. En estos sistemas, la ausencia del SE de regulación de la erosión del suelo posibilita la provisión de alguno de sus SE culturales. El objetivo de este trabajo es valorar socioeconómicamente, mediante un ejercicio de valoración contingente, medidas de gestión para proteger los Badlands de la Región de Murcia. Se ha realizado una encuesta a 289 hogares que ha permitido evaluar la percepción de la sociedad regional del fenómeno de la erosión, la importancia de los SE de estos espacios y la valoración de las medidas de gestión planteadas. Entre los resultados destaca el elevado número de respuesta protesta y una disposición media al pago de 15,93 €/hogar año por las medidas de gestión, entre las que limitar la expansión de la agricultura es percibida como la más eficaz.

Palabras clave: Servicios ecosistémicos, Valoración contingente, Medidas de gestión.

\section{Introducción}

En zonas áridas y semiáridas existen ecosistemas muy erosionados que se caracterizan por una baja provisión de servicios ecosistémicos (SE) de abastecimiento (ej. Producción alimentos) y regulación (ej. Control de erosión). Asimismo estos espacios se vienen percibiendo en muchas ocasiones como carentes de SE culturales, cuando realmente la ausencia del SE de control de erosión del suelo permite ofrecer una variedad de SE culturales singulares (ej. Valor estético o actividades recreativas), como ocurre en el caso de los Badlands (Nadal-Romero y García-Ruiz, 2018).

Los Badlands son ecosistemas erosionados en barrancos y cárcavas que disponen de sedimentos y regolitos poco consolidados, escasa vegetación y un drenaje intenso. Estos ecosistemas son resultado de factores físicos y climáticos, y en algunos casos, de la actividad humana, lo que da lugar a una diversidad de formas y paisajes característicos de la zonan en que se localicen (Belmonte-Serrato et al., 2019).

En España, se han aplicado figuras de protección en algunos de estos ecosistemas como es el caso de Parques-Parajes Naturales y Paisajes Protegidos (ej: Bardenas Reales, Desierto de Tabernas y los Barrancos de Gebas), lo cual es indicativo del valor cultural y natural que poseen estos espacios. No obstante, el desconocimiento de la población junto a la falta de interés de algunas administraciones ha permitido una elevada presión antrópica en muchos de estos espacios (Zgłobicki et al., 2019).

El objetivo de este trabajo ha sido determinar el valor socioeconómico de medidas de gestión que protejan de la presión antrópica los Badlands de la Región de Murcia (Mapa 1), donde la expansión de la agricultura y las actividades a motor, entre otros, amenazan su conservación. 
Mapa 1. Localización de los Badlands de la Región de Murcia.

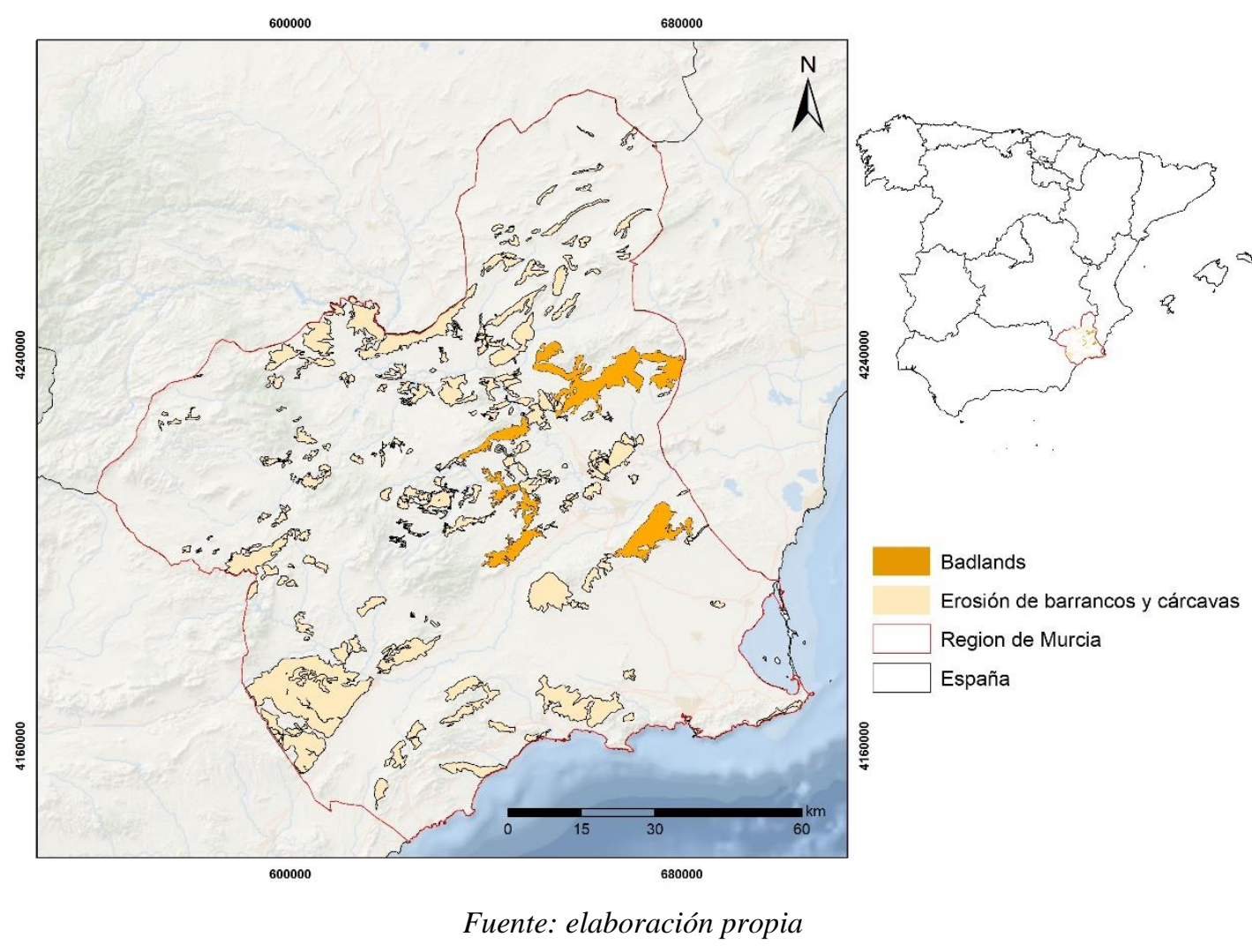

\section{Materiales y métodos}

En este trabajo se han aplicado metodologías de participación social y de valoración económica, utilizando encuestas directas a la población de la Región de Murcia.

Mediante escalas de Likert decimales (de 0 a 10) y técnicas de comparación por pares, se obtuvo la percepción social de aspectos relacionados con: (I) la importancia que tiene la pérdida del suelo, tanto en zonas fértiles como en las zonas erosionadas; (II) los SE que se deberían potenciar en zonas erosionadas; y (3) la aplicación de medidas de gestión que permitan proteger los Badlands de la Región de Murcia. Para la valoración económica de las medidas de gestión se utilizó el método de valoración contingente, dada la ausencia de mercado para los servicios a evaluar.

El proceso de encuesta se llevó a cabo de forma personal en el mes de diciembre de 2020, siendo la población objetivo los 542.000 hogares de la Región de Murcia. La muestra obtenida (289 encuestas) proporciona un error muestral del 5,8\% en proporciones intermedias y del 3,5\% en proporciones extremas, a un nivel de confianza del $95 \%$ para una variable dicotómica.

\section{Resultados}

La descriptiva de la muestra indica que, en promedio, el encuestado es de nacionalidad española, con un nivel de estudios medio-alto, y una edad media de 39 años. Lo más frecuente es un hogar de 4 miembros, siendo el tamaño medio familiar de 3,3 personas, con una renta familiar media de $1.963 € / \mathrm{mes}$. Estos valores permiten afirmar la representatividad de la muestra, dado que no difieren de los rasgos poblacionales de la Región de Murcia (CREM, 2020).

\subsection{Percepción del fenómeno de la erosión y valoración de medidas de gestión}

Los encuestados señalaron que la pérdida del suelo fértil (ej. Agricultura) es un fenómeno más importante que la pérdida de zonas singulares (ej. Badlands). Mediante una comparación por pares entre los tres principales tipos de SE (abastecimiento, regulación y culturales) y utilizando la técnica Analytic Hierarchy Process (AHP), se obtuvo la importancia relativa dada a los SE que se deben potenciar en espacios 
erosionados de la región (Cuadro 1). La población indica que se deben potencien los SE de regulación (38\%), seguido de los culturales (32\%) y abastecimiento (30\%). Además, la asociación entre las características socioeconómicas y las preferencias de SE mostró varias relaciones significativas (p.valor < $0,10)$ de interés, recogidas también en el Cuadro 1.

Cuadro 1. Importancia relativa de los servicios ecosistémicos a potenciar en espacios erosionados según características socioeconómicas

\begin{tabular}{lccc}
\hline \multicolumn{1}{c}{ Variable (n) } & SE Cultural & SE Regulación & SE Abastecimiento \\
\hline Total (289) & 0,32 & 0,38 & 0,30 \\
\hline Renta alta [>3.000 €] (29) & 0,46 & 0,35 & 0,19 \\
Renta media [1.000-3.000 €] (195) & 0,32 & 0,37 & 0,31 \\
Renta baja [<1.000 €] (65) & 0,26 & 0,43 & 0,31 \\
\hline Sin trabajo (125) & 0,33 & 0,35 & 0,32 \\
Con trabajo (164) & 0,31 & 0,40 & 0,29 \\
\hline Pérdida suelo zonas singulares (118) & 0,44 & 0,37 & 0,19 \\
Pérdida suelo zonas fértiles (171) & 0,24 & 0,39 & 0,37 \\
\hline Sin estudios universitarios (145) & 0,30 & 0,40 & 0,30 \\
Con estudios universitarios (144) & 0,34 & 0,36 & 0,30 \\
\hline Jóvenes [18-30 años] (122) & 0,37 & 0,34 & 0,29 \\
Adultos [30-60 años] (139) & 0,29 & 0,41 & 0,30 \\
Ancianos [>60 años] (28) & 0,25 & 0,42 & 0,33 \\
\hline
\end{tabular}

Fuente: elaboración propia.

Así, las familias que poseen una renta más alta y son más jóvenes creen que se debe de potenciar los SE culturales, disminuyendo la importancia relativa dada a las otras dos categorías. Los encuestados que consideraron más importante la pérdida de zonas singulares como los Badlands respecto a la pérdida de zonas agrícolas muestran también una preferencia clara por los SE culturales. En cambio, para los que consideraron más importante la pérdida del suelo agrícola, potenciar los SE de regulación y de abastecimiento ha de ser prioritario. Por último, las personas que son trabajadoras activas y no tienen estudios universitarios mostraron una mayor preferencia por potenciar los SE de regulación.

A continuación, a los encuestados se les pidió que valoraran de 0 a 10 la efectividad que podía tener la aplicación de medidas de gestión con la finalidad de proteger los Badlands (Cuadro 2), entre las cuales, las más demandadas estaban relacionadas con limitar la expansión agrícola sobre estos ecosistemas y prohibir construcciones y actividades a motor.

Cuadro 2. Valoración de la efectividad de medidas de gestión

\begin{tabular}{lc}
\hline \multicolumn{1}{c}{ Medida } & Valor \\
\hline Limitar la expansión de la actividad agrícola & 8,22 \\
\hline Limitar las reforestaciones & 6,23 \\
\hline Prohibir actividades deportivas a motor (motos, quads...) & 8,00 \\
\hline Prohibir las construcciones (residenciales, carreteras y caminos, industriales...) & 8,16 \\
\hline Promocionar los valores socio-ambientales (itinerarios, rutas turísticas...) & 7,82 \\
\hline
\end{tabular}

Fuente: elaboración propia

\subsection{Valoración económica de las medidas de gestión}

Una vez valoradas individualmente las medidas de gestión se preguntó a los encuestados por la disposición a pagar (DAP) para que se llevaran a cabo dichas medidas. El vehículo de pago, seleccionado en la encuesta piloto, es una cuota anual a una asociación sin ánimo de lucro (ej. asociación para la custodia del territorio). Del análisis de respuestas del ejercicio de valoración contingente se desprendió que, de las 289 encuestas, el 34,60 \% de la muestra (100 individuos) mostraban una DAP $>0$, mientras que el $65,40 \%$ (189 individuos) restante no estarían dispuestos a pagar por las medidas (DAP=0). Para distinguir a los encuestados que 
realmente no están dispuestos a hacer una aportación ya que no valoran estos ecosistemas (los ceros reales) y aquellos que su negativa al pago expresa su rechazo al mercado propuesto en la encuesta (ceros protesta), se les preguntó por las razones de su $\mathrm{DAP}=0$, siendo el argumento más frecuente que la mejora y conservación de los Badlands debería correr a cargo de los presupuestos públicos. Esto llevó a concluir que 128 de los 189 encuestados con una nula DAP se correspondían con ceros protesta, siendo los 61 restantes los ceros reales. El mercado hipotético se conforma con los encuestados de DAP $>0$ y los ceros reales, y queda finalmente compuesto por 161 hogares.

La DAP de cada hogar de la Región de Murcia por la aplicación de medidas de gestión para la protección de los Badlands ha sido, en media, de 15,93 €/año, con un mínimo de 0 y un máximo de $100 € /$ hogar año. A partir de la DAP media individual se estimó la DAP agregada al conjunto de la población afectada, es decir, el Valor Económico Total, que para el conjunto de hogares de la Región de Murcia es de aproximadamente 8.634.060 €/año.

\section{Conclusiones}

Este trabajo ha permitido realizar una primera aproximación a la valoración socioeconómica de un ecosistema muy poco estudiado en la literatura económica, los Badlands. Los mismos son zonas singulares de algunas zonas áridas, como es el caso de la Región de Murcia, que tienen entre sus orígenes la ausencia de un SE de regulación como es el control de la erosión del suelo.

La población murciana, en general, considera que en estas áreas deberían de potenciarse los SE de regulación. Aquellas personas que consideran que es más importante la pérdida de la agricultura que la de estas zonas singulares creen que los SE de regulación y abastecimiento deben de ser los que más se potencien en espacios erosionados. Por el contario, los encuestados que otorgan una mayor importancia a los Badlands, consideran que se debe de potenciar los SE culturales, como son la estética del paisaje o las actividades recreativas.

Las medidas de gestión propuestas recibieron una puntuación media notable en su conjunto, y a pesar de las preferencias de una parte de la población por la agricultura, consideraron que limitar la expansión de esta actividad sobre los Badlands es clave para su protección y conservación.

El ejercicio de valoración económica mostró un elevado número de respuestas protestas $(44,29 \%$ de la muestra) y un VET de 8,6 millones de $€ /$ año por aplicar medidas de gestión que protejan los Badlands. Este tipo de información es de utilidad para los encargados de la toma de decisiones sobre planificación territorial y gestión de los espacios, con la finalidad de que las actuaciones a realizar sean socialmente aceptadas.

\section{Agradecimientos}

Este trabajo se ha realizado en el marco del proyecto 20912/PI/18 financiado por la "Fundación SénecaAgencia de Ciencia y Tecnología de la Región de Murcia" y por el MECD (FPU16/03562).

\section{Bibliografía}

Belmonte-Serrato, F., Romero Díaz, A. y Ballesteros Pelegrín, G. A. (2019). "Los paisajes áridos abarrancados como recurso turístico". Investigaciones Turísticas, 17: 213-238.

CREM (2020). Centro Regional de Estadística de Murcia. Datos municipales. Disponible en: https://econet.carm.es/web/crem/inicio/-/crem/sicrem/PU_datosBasicos/Indice1.html

Nadal-Romero, E., y García-Ruiz, J. M. (2018). Rethinking Spatial and Temporal Variability of Erosion in Badlands. En Nadal-Romero, E. Martinez-Murillo, J. F. y Kuhn, N. (Eds): Badlands Dynamics in a Context of Global Change. Elsevier: 217-253.

Zgłobicki, W., Poesen, J., Cohen, M., Del Monte, M., García-Ruiz, J. M., Ionita, I., y Pica, A. (2019). “The potential of permanent gullies in Europe as geomorphosites". Geoheritage, 11(2): 217-239. 